\title{
Moving minds: Mental health and wellbeing benefits of a 50-day workplace physical activity program
}

\author{
Karen T. Hallam ${ }^{1,2}$ (D) Anna Peeters ${ }^{3}\left[\right.$ - A. Gupta ${ }^{3} \cdot$ S. Bilsborough ${ }^{2}$ \\ Accepted: 14 November 2021 \\ (c) The Author(s), under exclusive licence to Springer Science+Business Media, LLC, part of Springer Nature 2021
}

\begin{abstract}
The aim of this study was to assess changes in mental health and wellbeing measures across a 50-day physical activity workplace program. The secondary aims assessed the relationship between demographic and pre-program physical activity self-reported variables, mental health, wellbeing and program engagement measures. The study utilized a naturalistic longitudinal design with a study population of 2903 people. Participants were engaged in the 10,000 step daily physical activity program for 50-days and measures of engagement were tracked. 1320 participants provided full pre/post-program data across a range of standardized mental health and wellbeing measures alongside demographic and program engagement measures. For individuals providing pre and post program data there was a significant reduction in anxiety $(18.2 \%, \mathrm{p}=.008)$, stress $(13.0 \%, \mathrm{p}=.014)$ and sleep related impairment $(6.9 \%, \mathrm{p}<.001)$ alongside a significant improvement in overall wellbeing $(6.7 \%, \mathrm{p}=.001)$. The data further showed no significant mental health differences were identified between individuals who recorded below versus equal to or above 10,000 steps. Regression analyses indicated numerous group and personal variables impacted mental health, wellbeing and program engagement. The study highlights improvements in a range of mental health and wellbeing scores occurred over the 50-day activity program for people who complete the program. Finally, the study identified a range of protective and risk factors for mental health benefits of these programs and level of engagement. Whilst there were similarities in the pre-program mental health and wellbeing scores of those who completed and those lost to follow-up, further research is required to better characterize and understand this group.
\end{abstract}

Keywords Mental health $\cdot$ Wellbeing $\cdot$ Physical activity $\cdot$ Workplace $\cdot$ Activity-tracking $\cdot$ Intervention

\section{Introduction}

Australia is experiencing an emerging mental health crisis along with the wider global community. Mental illness now impacts $45 \%$ of the Australian population at some stage of their lives and $20.1 \%$ of Australians in 2017 (Australian Bureau of Statistics, ABS , 2018). The World Health Organization has indicated that mental illnesses like depression will be the leading cause of disease burden globally by 2030

Karen T. Hallam

Karen.Hallam@deakin.edu.au

1 Institute for Mental and Physical Health and Clinical Translation, School of Medicine, Deakin University, PO Box 281, Geelong, Vic 3220, Australia

2 Moving Mindz Pty Ltd, Melbourne, Australia

3 Global Obesity Centre, Institute for Health Transformation, School of Health and Social Development, Deakin University, Geelong, Australia
(World Health Organization, 2011). This increase in disease burden is associated with significant personal, family, workplace and community distress (Doran \& Kinchin, 2019). The Australian Institute of Health and Welfare (AIHW) highlight that depression and anxiety are two of the ten leading causes of burden of disease in Australia (AIHW , 2016). As the Australian annual cost of mental illness passes $\$ 50$ BN and \$180 BN in lost productivity (Productivity Commission, 2019), strategies increasingly need to focus on illness prevention and improving modifiable risk factors of mental health issues through simple and evidence-based approaches.

The majority of the world's population (58\%) spend one third of their life at work (WHO, 1994). Mental illness has an enormous economic toll on Australian workplaces, costing \$12.8 billion annually (KPMG and Mental Health Australia, 2018) through absenteeism, presenteeism and work claims. These impacts are not only associated with clinically diagnosed depressive and anxiety illnesses 
(Bailey et al., 2016), but also with psychological distress (often called sub-syndromal symptoms). For example, high levels of stress (i.e. distress) have been linked with both increased absenteeism and presenteeism (Coutu et al., 2015; Holden et al., 2011). Notably, moderate levels of psychological distress are more prevalent than clinical levels in the workforce yet contribute to workplace accidents and lower levels of worker success (Hilton \& Whiteford, 2010). These data reinforce the emerging awareness that workplaces have regarding the synergy between mental health and wellbeing and workplace performance (Robertson \& Cooper, 2010). Fortunately, workplaces are also an ideal microcosm to improve mental health and wellbeing in their workforce.

Physical activity has long been recognized for its benefits on physical health and reducing the incidence, severity and impacts of numerous non-communicable diseases (Reiner et al., 2013). In a landmark paper, Booth explains that physical activity/exercise is examined as primary prevention against 35 chronic conditions including mental health (Booth et al., 2012). This work extended into the mental health arena as early as 2001, when theories of the mechanism of action of exercise on mood were being proposed (Salmon, 2001). These theories highlight not only the immediate endorphine related impacts of exercise on mood, but also chronic improvements in mood that were likely independent from aerobic fitness levels. These improvements included both antidepressant and anxiolytic effects alongside reducing sensitivity to stress (Salmon, 2001). In clinical settings, exercise has been shown to improve symptoms of depression (Schuch et al., 2016) and anxiety (Stubbs et al., 2017a). Moreover, the evidence accumulated for the positive impacts of exercise now place the intervention equal to medication and psychotherapies for moderate depressive disorders (Ekkekakis \& Murri, 2017). Research into psychological distress in the workplace indicates that physical activity is associated with resilience to stress. Conversely, individuals reporting high levels of stress and more mental health issues were less likely to exercise (Gerber et al., 2014). A meta-analysis by Abdin et al. (2018) of four papers (three high quality) on walking interventions showed improvements in vitality, work performance and reduced fatigue at four months follow-up. Importantly, walking interventions were most indicated in sedentary workers. Physical activity is only one of the workplace mental health approaches available in many workforce programs. Joyce et al. (2016) conducted a meta-analysis of 20 high quality reviews on workplace interventions for mental health issues. Their outcomes highlight physical activity as one of only two, workforce primary prevention approaches that improved mental health in workers (alongside enhancing employee sense of control). Despite indicating moderate evidence from their analysis, these authors highlighted that more research is needed to assess which type, amount and intensity of exercise is optimal.

Workplace activity-tracking physical activity programs provide low cost and highly engaging 'program' based activities to promote engagement in physical activity, irrespective of intensity (Sounan et al., 2013). Walking is the most accessible physical activity and most workers are capable of engaging with walking despite socio-economic background, skill and commencing fitness levels (Aittasalo et al., 2012). The general goal of walking 10,000 steps daily has been widely adopted by the fitness industry, fitness tracking devices and the population in general (Brody \& Burn, 2015). Notably, peer reviewed evidence also indicates positive health benefit for body composition, lipid levels and blood pressure improvements (Wattanapisit \& Thanamee, 2017) occur with this level of activity.

Activity-tracking interventions likely exert their impact through motivational processes, namely the process of goal setting, which is particularly successful if done in groups and goals announced to others (Epton et al., 2017), and self-monitoring of progress (Sanders et al., 2016). These 'programs' also imbue evidence-based behaviour change stimuli of gamification (Johnson et al., 2016) and team engagement, which decreases dropout and increase activity levels (Glance et al., 2016). These components fit well into the Health Action Process Approach (HAPA, Schwarzer, 2008) behaviour change theory that postulates health behaviours are elicited by the combination of motivational factors (workplace and staff promotion and enthusiasm) and goalpursuit phase (in this case the setting and achieving of step and team goals and mini-programs).

Workplace based physical activity programs may play a role in improving mental health and wellbeing. FreakPoli et al. (2014) have demonstrated marked improvements in wellbeing after a four-month activity-tracking-based program with sustained results at eight-month follow-up alongside improved health related quality of life (Harding et al., 2013). Sounan et al. (2013) demonstrated significant improvements in stress, fatigue and sleep disturbance scores after an even briefer 8-week, 10,000 step, activity-tracking workplace-program. The study showed a strong retention rate (over 75\%) and a range of physical health benefits. A study reporting on 1-year follow-up of a workplace health program integrating an eight-week activity-tracking-based program provides good insights into the maintenance and sociodemographic influencers in activity-tracking interventions. In this study, Lowensteyn et al. (2018) articulated their use of motivational and goal pursuit elements using a gamification approach. This program included engaging people in teams, nominating leaders, goalsetting, message board, e-mail reminders and physical incentives. Step goals were adjusted through the program via programs. Noting that the intervention was one of a range of interventions to support 
worker health and wellbeing, the outcomes show significant improvements in sleep quality, emotional stress and fatigue scores at 12-months follow-up. Our own research found similar improvements in mental health measures after a 100-day activity-tracking program (Hallam et al., 2018). This study focused more specifically on mental health and wellbeing outcomes with measures of depression, anxiety and stress alongside overall wellbeing being assessed (Hallam Bilsborough and de Courten). The results showed significant improvements in psychological distress scores on depression, anxiety and stress measures after a 100-day activity program (just over 14 weeks). Notably, these effects were independent of whether individuals reached a 10, 000 step goal, although there were statistically significant increase step counts across the course of the program.

The aims of this study were to both characterise engagement in the workplace physical activity program and follow-up the mental health outcomes for the individuals who provided both pre and post-program survey results. The first hypothesis explored the level of engagement with the program. This analysis included identifying baseline differences in pre-event mental health findings across participants who completed the program and follow-up questionnaires, those who engaged in the program but did not complete the post-event mental health scales (lost to follow-up group) and those that dropped out of the program.

In relation to the group of program completers, based on our previous findings of mental health improvements after a 100-day physical activity program, combined with biological evidence that exercise mental health benefits of exercise begin within 8-weeks, it was hypothesised that mental health and wellbeing benefits may begin to occur over 50-days of a physical activity program. Our previous findings (Hallam et al., 2018) also highlighted that reaching 10,000 steps had little impact on outcomes, so in this study we again assessed the impacts of reaching this threshold. The final hypotheses from the study focused on whether socio-demographic factors impacted mental health and wellbeing outcomes including depression, anxiety, stress, sleep disturbance, sleep impairment and general wellbeing.

\section{Method}

\section{Participants}

This study included a total of 2903 individuals (879 men, 1980 women and 45 people who chose not to indicate gender). All participants were employees from organizations across Australia and New Zealand who were participating in the workplace physical activity program. Retrospective ethics approval to analyse the results of the sample collected between October and December 2019 was obtained from Deakin University Human Research Ethics Committee (HREC 2019-381).

\section{Materials}

\section{Demographic and Subjective Pre-Program Activity Level Questionnaire}

The pre-event questionnaire collected demographic data around age, gender, marital-status and number of children. Self-report health behaviours prior to the intervention were recorded including: an estimate of the number of hours spent sitting daily and an estimate of the number of steps they believe they are doing daily at the commencement of the program (categorically rated as between 0 and 4999 steps, 5000-7499 steps, 7500-9999 steps, 10,000-12,499 steps, 12,500 and above steps).

\section{Program Participation Outcomes}

New categorical variables were calculated to indicate engagement with the program. These included a calculation of the average number of steps completed daily (based on total number of steps recorded/ total number of days steps recorded in App) and number of days steps recorded across program (number of days steps recorded in App over the fifty-day period). Individuals were identified as 'dropping out' if they enrolled in the program and recorded their activity on less than or equal to $10 \%$ (five days) of the 50-day program. Individuals were identified as lost to follow-up if they completed more than $10 \%$ of the program but did not complete the post-event questionnaires.

\section{Depression, Anxiety and Stress Scale (DASS-21)}

The Depression, Anxiety and Stress Scale (DASS-21) is a 21-item self-report measure of symptoms of depression, anxiety and stress, developed in Australia and used internationally to measure psychological distress (Lovibond \& Lovibond, 1995). The scale is not diagnostic, it rather highlights the level of symptomatology. The DASS-21 is the short form of the DASS-42 and has seven questions on each of three sub-scales that report on depressive, anxiety and stress symptoms. To complete, individuals rate their experience on a four-point scale from 0 (did not apply to me at all) to 3 (applies very much to me/most of the time). The DASS has strong construct validity (Crawford \& Henry, 2003), adequate discriminant and convergent validity (Lovibond \& Lovibond, 1995) and internal consistency (Cronbach's $\alpha$ of $0.96,0.89,0.93$ for the depression, anxiety and stress subscales respectively) (Brown et al., 1997). It has strong factor structure in both non-clinical (Lovibond \& Lovibond, 1995) and clinical samples (Brown Chorpita Korotitsch and 
Barlow). All DASS-21 scores were multiplied by 2 to provide a score comparable to the original DASS-42 (Lovibond \& Lovibond, 1995).

\section{PROMIS Sleep Disturbance and Sleep Impairment Scale}

The Patient Reported Outcomes Measurement Information System (PROMIS) was developed by the National Institute of Health (see www.nihpromis.org) in the U.S.A and is used extensively in international research. In Australia, recent national guidelines related to sports medicine recommend the use of this measure for assessing clinical experiences in those with cancers (Hayes et al., 2019) and the measure has also recently been used in Australian mental health populations (Strainge et al., 2019) and shows similar outcomes to objective sleep quality measures in research on stress and sleep in Australian populations (Yap et al., 2020). PROMIS provides a series of measures to improve patient outcome recording across physical and psychological domains. The PROMIS Sleep Disturbance (PROMIS-SD) and PROMIS Sleep Related Impairment (PROMIS-SRI) Scales were developed using item response theory to increase psychometric soundness. The PROMIS-SD scale measures disturbance in quality including initiation and sustenance of quality sleep. The eight items were measured on a scale from 1 (not at all) to five (very much) and are summed (the "my sleep was refreshing' item was first reversed) to provide a total sleep disruption score. The eight item PROMIS-SRI reports on daytime impairment related to sleep disturbance. The scale is rated from 1 (not at all) to 5 (very much). The item 'I felt alert when I woke up" was reverse scored and the items were summed to provide a total score on the measure. Research indicates that the PROMIS short forms have strong internal consistency and reliability and greater measurement precision against the Pittsburgh Sleep Quality Index and the Edinburgh Sleep Scale (Yu et al., 2011).

\section{WHO-5 Wellbeing Index}

The WHO-5 Wellbeing Index (English version) is a brief (5-item) subjective quality of life measure comprising items related to positive mood, vitality and levels of interest. The index is anchored on a six-point scale from 5 (all the time) to 0 (at no time). Scores are summed to provide a raw score between 0 and 25 , which is then multiplied by four to provide a score out of a possible 100 , with 100 being excellent wellbeing. The WHO-5 is a strong measure for assessing the general factor of wellbeing (Hall et al., 2011). A systematic review of over 200 trials indicates the measure is both valid as a screening tool as a general measure of wellbeing (Topp et al., 2015). The WHO-5 has been used extensively internationally in research settings and is one of the most widely used wellbeing measures that has been made available in over 30 languages (Topp et al., 2015). This study utilises the most widely used the WHO-5 (English) version.

\section{Procedure}

The study reports on the outcomes of the Moving Mindz program. This program is an online workplace-based program that encourages physical activity through teams working towards increasing walking and physical activity. Organizations participate in the program and within organizations, individuals are encouraged to participate individually or in teams of up to five members. Organizations may incentivise the teams by providing prizes for distances walked and other programs, but this is not provided by the program itself. The program encourages participants to aim for the typical 10,000 step goal as has been used in previous research and commercial applications. Step activity is logged by individuals using their existing personal mobile phone or physical activity monitoring device (typically wrist-worn fitness trackers). Participants regularly $\log$ their results into the online step portal from their devices recordings (preferably daily) to provide their own and their teams' progress in the program. Reminders are sent to participants regarding logging step counts regularly. Throughout the program, participants are provided with feedback on their step progress, individual and group leader boards within the organization, mini-challenges and brief psycho-education e-mails on the mental and physical health benefits of physical activity to increase participation and retention. Further information on the program can be obtained from https://movingmindz.io/or the corresponding author.

Participants enrolled in the workplace program between either 7-10-2019 to 29-11-2019 or 16-10-2019 to 7-12-2019 were offered the opportunity to participate in the current research project. Participation was voluntary, anonymous to their organization (i.e., the identity of study participants was not provided back to organizations) and participants were not reimbursed for taking part in the study. To participate in the study, individuals provided informed consent and completed a pre and post-event questionnaire via their online program App and recorded all program steps and activities as usual. The pre-event questionnaire including sections relating to demographic and pre-program physical activity levels. In addition to standard demographic questionnaires, participants were asked to complete numerous validated mental health and wellbeing measures pre-program and post-program.

Upon completion of the 50-day program, participants were provided a five-day window to complete the post-event questionnaires. At the end of the program, participants were provided with a form fill standard feedback form prepared by a clinical psychologist based on their results on the scales. This feedback provided general information about depression, anxiety, stress and wellbeing and ranked their results by category (i.e. low, moderate and high/very high). Also included were 
contact details for services and supports within Australia, if their scores fell within the "very high" range on the mood and anxiety measure.

\section{Study Design and Data Analysis}

This study utilised a naturalistic longitudinal study design. The sample was opportunistic and comprised consenting individuals already enrolled in a workplace sponsored wellbeing program. Participants from the larger cohort were invited to complete the mental health assessments with the knowledge that this would be used for direct feedback purposes to individuals and for research purposes (self-selected opportunistic sample). For individuals who consented to being involved in the research, data were transferred from the online platform into SPSS version 26 for statistical analysis, where identifying data was immediately removed and replaced with a de-identified research study number. String data were coded into nominal categories (e.g. team names became a unique team identifier) and results on the outcome questionnaires calculated using the approaches described in the original papers included in the material section.

All data were assessed for normality and outliers. Outliers were removed from the dataset if their scores lay more than two standard deviations from the population mean (see Field, 2018 for discussion). Normality was assessed using the Shapiro-Wilk method. Descriptive statistics were generated to characterise the cohort followed by inferential statistics. The exploratory hypothesis related to program engagement was explored using descriptive statistics and differences between groups (program completers, dropouts and those lost to follow-up) were assessed across the mental health and wellbeing scales using independent sample ANOVA's. The hypothesis regarding mental health outcomes for program completers was assessed using paired samples ANOVA's on the mental health and wellbeing outcomes. The hypothesis investigating the impact of reaching the 10,000-step threshold was also analysed using an ANOVA. Bonferroni corrections were not used on these hypotheses tests as per peer-reviewed recommendations (see Perneger, 1998 for full discussion). The final hypothesis utilised multiple regression to investigated the relationship between predictor variables of age, gender, number of children, self-reported pre-program step average and self-reported pre-program estimated sitting duration on post-program mental health and wellbeing outcomes.

\section{Results}

\section{Demographics of Overall Cohort}

An original study population of 2903 individuals consented to participate in the research study $(878 \mathrm{M}, 1981 \mathrm{~W}$ and 45 preferred not to specify) and completed the pre-event surveys. Age ranged between 18 and 81 years with the average being 38.9 years $($ S.D. $=11.6)$. Most individuals participating were single $(30.2 \%)$ or married/partnered $(52.4 \%)$ with smaller numbers divorced $(4.7 \%)$, widowed $(0.8 \%)$ or preferring not to disclose (11.9\%). Almost half of the sample did not have children $(46.2 \%)$ with a further $12.3 \%, 26.1 \%$, $8.0 \%, 1.9 \%, 0.8 \%$ and $4.8 \%$ having one, two, three, four, five and six children respectively. Team sizes were recorded and indicated that most individuals competed in teams of five (53.9\%) or four $(27.5 \%)$ team members. Fewer individuals competed in groups of three (12.6\%), two $(4.5 \%)$ or alone $(1.2 \%)$.

\section{Program Engagement Outcomes}

Program engagement was measured by assessing the number of days that steps were logged and the average number of steps recorded daily. Fig. 1 highlights the number of days that steps were logged and demonstrates a very low rate of program dropout (defined in this research as recording step counts for $10 \%$ or less of the program (i.e., 5 days or less). Moreover, most participants $(63.3 \%)$ recorded steps for at least $80 \%$ of the program ( 40 out of 50 days), showing a high rate of engagement.

The second measure of engagement included measuring average step numbers amongst the complete cohort based on the average steps for all days that steps were recorded. The outcomes highlight a wide range in average step counts from 1128 to 51,853 . The average number of steps completed in the overall cohort during the activity program was 12,095 (S.D. $=5033$ ).

\section{Assessing Pre-Program Differences between Program Completers, Dropouts and those Lost to Follow-up in Mental Health and Wellbeing Measures}

The analysis of the database indicated high rates of program completers $(n=1320)$, and individuals lost to follow-up $(n=1374)$ with lower levels of program dropouts $(n=209)$. Due to high rates of individuals lost to follow-up, univariate ANOVA's were performed to assess pre-program differences in mental health and wellbeing profiles of these cohorts to establish whether further analysis of the program completer group was valid or if the mental health characteristics of this group was significantly different. The data for this analysis are presented in Table 1.

The outcomes of Table 1 indicate that there were few significant differences observed in the mental health and wellbeing status of individuals who completed the program, those who dropped out during the program and those lost to follow-up. The only two significant findings were associated with decreased anxiety and higher wellbeing scores in 


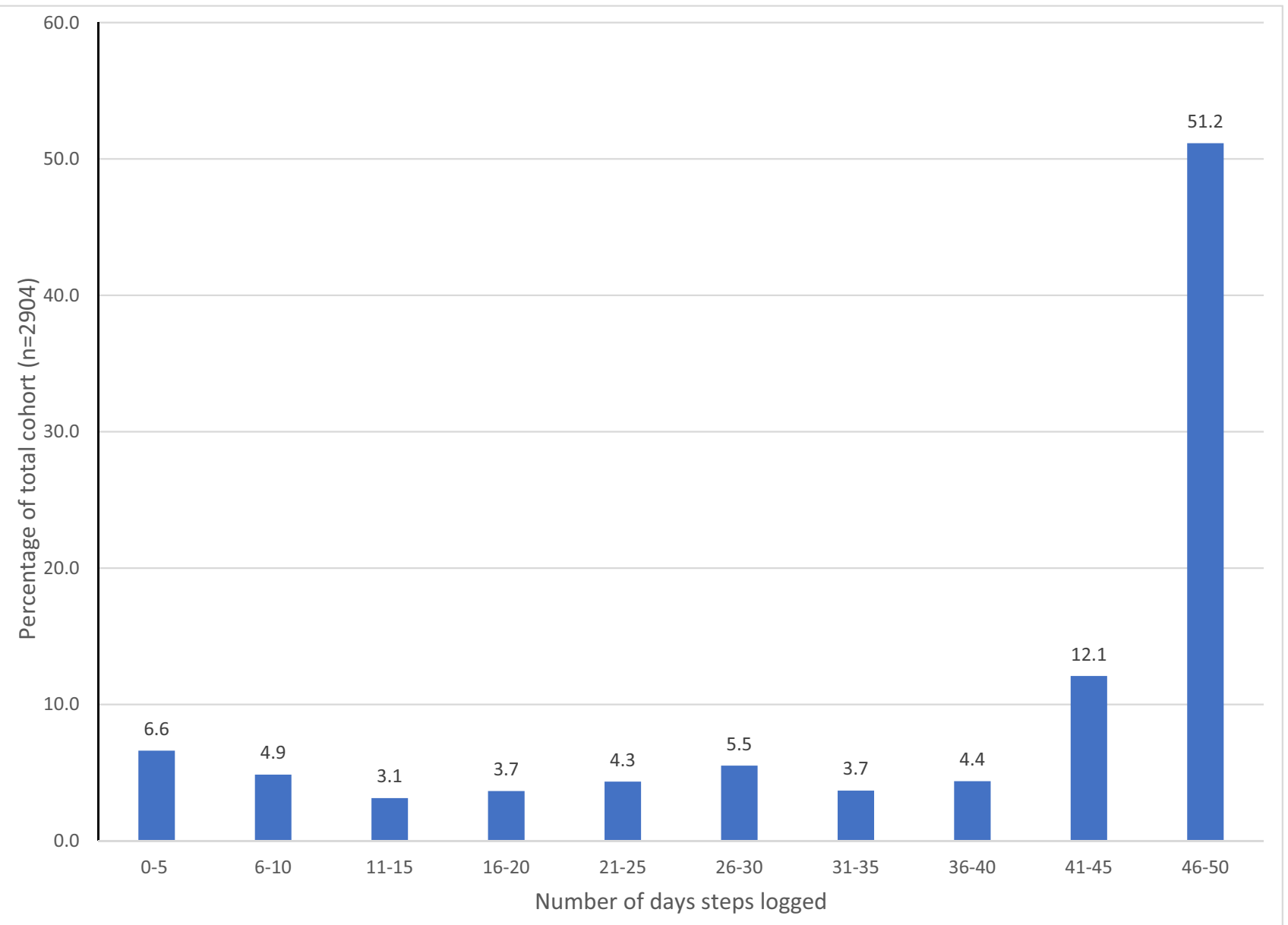

Fig. 1 Number of days steps logged across program for all program participants

program completers versus those lost to follow-up. Analysis of effect size estimates indicates a very modest difference between these groups. Overall, these data indicate the mental health and wellbeing profile of individuals within the program completer, dropout and lost to follow-up groups were very similar, indicating analysis of the former group may reflect a reflective sub-set of the larger sample.

\section{Mental Health and Wellbeing Changes across Time in Program Completers}

Of the 1324 program completers, the analysis sample included 434 men, 869 women and 21 participants who preferred not to specify. The average age of completing participants was 39.9 years $($ S.D. $=11.7)$ with a range between

Table 1 Mental health and wellbeing difference between program completers, drop-outs and those lost to follow-up

\begin{tabular}{|c|c|c|c|c|c|c|}
\hline & DASS Depression & DASS Anxiety & DASS Stress & WHO-5 & PROMIS SD & PROMIS SRI \\
\hline $\begin{array}{l}\text { Program Com- } \\
\text { pleters }\end{array}$ & $6.11(6.52)$ & $5.36(5.32)$ & $10.13(6.70)$ & $73.45(21.77)$ & $3.12(.52)$ & $2.96(.86)$ \\
\hline Drop-out & $6.44(6.51)$ & $6.15(5.93)$ & $10.67(6.87)$ & $70.02(22.17)$ & $3.16(.52)$ & $2.99(.81)$ \\
\hline Lost to follow-up & $6.81(9.03)$ & $6.04(8.18) *$ & $10.39(9.08)$ & $68.88(27.70)^{*}$ & $3.09(2.81)$ & $3.08(2.88)$ \\
\hline Statistics & $\begin{array}{l}F(2,2902)=2.72 \\
p=.066 \\
\text { eta }=.002 \\
\text { power }=.539\end{array}$ & $\begin{array}{l}F(2,2902)=3.62, \\
\quad p=.027 \\
\text { eta }^{2}=.002 \\
\text { power }=.671\end{array}$ & $\begin{array}{l}F(2,2902)=630 \\
p=.533 \\
\text { eta }{ }^{2}=.000 \\
\text { power }=156\end{array}$ & $\begin{array}{l}F(2,2902)=11.645 \\
p<.001 \\
\text { eta } \\
\text { power }=.008 \\
\end{array}$ & $\begin{array}{l}F(2,2902)=.154 \\
\quad p=.858 \\
\text { eta }=.000 \\
\text { power }=.074\end{array}$ & $\begin{array}{l}F(2,2902)=.315 \\
p=.730, \\
\text { eta }{ }^{2}=.000, \\
\text { power }=.100\end{array}$ \\
\hline
\end{tabular}

*indicates significant differences in pre-program anxiety and WHO wellbeing levels between the program completer group and lost to follow-up group in post-hoc LSD testing 
18 and 81 years. Most individuals participating were single $(29.1 \%)$ or married/partnered $(54.3 \%)$ with smaller numbers divorced $(5.3 \%)$, widowed $(1.0 \%)$ or preferring not to disclose $(10.3 \%)$. Almost half of the sample did not have children $(42.2 \%)$ with a further $11.7 \%, 28.8 \%, 9.4 \%, 2.6 \%$ $1.2 \%$ and $4.1 \%$ having one, two, three, four, five and six children respectively. Team sizes were recorded and indicated that most individuals competed in teams of five $(63.9 \%)$ or four $(24.3 \%)$ team members. Fewer individuals competed in groups of three $(8.5 \%)$, two $(2.4 \%)$ or alone $(0.5 \%)$.

The outcomes for people completing the pre and post event surveys indicated they self-reported spending an average of $7.3 \mathrm{~h}$ daily sitting (S.D. $=2.7$ ) with a reported range of $0-20 \mathrm{~h}$ in the pre-event survey. Self-report of step activity pre-program revealed most of the cohort was sedentary with $8.4 \%$ indicating they completed less than 5000 steps daily and a further $32.7 \%$ and $26.3 \%$ indicating they completed between 5000 and 7499 steps and 7500 and 9999 steps respectively. A smaller proportion (16.7\%) completed over the recommended 10,000 steps prior to the program and $6 \%$ of the sample reported completing over 12,5000 steps daily. Of the participants who completed the program, the average number of steps (derived from step logs from each participant) showed that the majority of the cohort achieved an average of 10,000 or more steps daily $(n=934)$ compared with fewer participants who did not reach this benchmark $(\mathrm{N}=343)$. Overall, the average number of steps reported daily were 13,199 (S.D. =53) with a range between 3202 and 51,853 steps.

\section{Changes in Mental Health and Wellbeing over the Program}

A series of ANOVA were utilized to assess changes over time in the mental health and wellbeing measures. The results of these comparisons are presented in Table 2.

The outcomes of the mental health and wellbeing analyses indicate that anxiety, stress, wellbeing and sleep related impairment all significantly improved over the 50-day physical challenge in program completers. The scores on the study measures were already relatively low, highlighting the general positive mental health of the sample, the improvements in the scale scores were positive across the program with improvements in mental health scores of $9.8 \%$ for depressive symptoms (not statistically significant), $18.2 \%$ for anxiety symptoms and $12.0 \%$ for stress symptoms respectively. In terms of sleep disturbance and sleep related impairment, there was a pre to postprogram reduction in sleep disturbance and sleep related impairment of $1.9 \%$ (not statistically significant) and 6.9\% respectively. Finally, in relation to wellbeing there was a significant and marked improvement across the program period of $7.1 \%$.

\section{Impact of Reaching an Average of 10,000 Steps per Day on Mental Health and Wellbeing Measures}

Data were analysed based on program completers who reached an average of 10,000 steps or more daily versus those who did not reach this cut-off to assess whether reaching this criterion inferred any specific mental health and wellbeing benefits. The results of this analysis are presented in Table 3.

The associated ANOVA indicated that reaching 10,000 or more steps did not significantly impact on health and wellbeing measures, $F(1,1313)=3.60, p=.067$.

\section{Relationship between Basic Demographic and Pre-Program Self-Report Activity Levels with Mental Health and Wellbeing Outcomes in Program Completers}

A series of multiple regressions were performed to assess the impact of personal demographics (age, gender and number of children), team size and self-reported pre-program physical activity levels on mental health and wellbeing postprogram. The regression results are presented in Table 4.

Table 4 highlights that increasing age is associated with a range of improved mental health and wellbeing outcomes. Other demographic and team size factors did not significantly impact on post-program mental health and wellbeing
Table 2 Mental health and wellbeing changes from preprogram assessment time to post-program assessment time in program completers

\begin{tabular}{|c|c|c|c|}
\hline \multirow[t]{2}{*}{ Measure } & \multicolumn{2}{|c|}{$\begin{array}{l}\text { Estimated Marginal Mean } \\
\text { (S.E.) }\end{array}$} & \multirow{2}{*}{$\begin{array}{l}\text { Statistic } \\
\text { Statistic }\end{array}$} \\
\hline & Pre & Post & \\
\hline Depression & $6.55(0.49)$ & $5.91(0.50)$ & $F(1,1273)=3.139, p=.077, e^{2} a^{2}=.002$ \\
\hline Anxiety & $5.92(0.49)$ & $4.84(0.43)$ & $F(1,1273)=7.146, p=.008^{*}$, eta $^{2}=.005$ \\
\hline Stress & $9.96(0.46)$ & $8.76(0.51)$ & $F(1,1273)=6.10, p=.014^{*}, e t a^{2}=.005$ \\
\hline Wellbeing & $72.48(1.48)$ & $77.64(1.66)$ & $F(1,1273)=11.227, p=.001^{*}, e t a^{2}=.008$ \\
\hline Sleep Disturbance & $17.87(0.31)$ & $17.53(0.34)$ & $F(1,1273)=2.577, p=.109, e^{2 t a}=.002$ \\
\hline Sleep Related Impairment & $22.24(0.31)$ & 20.71(0.34) & $F(1,1273)=37.938, p<.001^{*}$, eta $^{2}=.028$ \\
\hline
\end{tabular}

$*$ denotes significant value at $\alpha=.05$ 
Table 3 Between subjects mean scores on mental health and wellbeing measures based on reaching 10,000 step threshold average daily (S.D.) in program completers

\begin{tabular}{|c|c|c|c|c|c|c|}
\hline & Depression* & Anxiety & Stress* & Wellbeing* & Sleep Disturbance* & $\begin{array}{l}\text { Sleep Related } \\
\text { Impairment }\end{array}$ \\
\hline$<10,000$ steps & $6.4(6.75)$ & $5.65(5.75)$ & $9.85(6.9)$ & $71.8(21.95)^{*}$ & $17.6(4.6)$ & $21.5(4.65)$ \\
\hline$\geq 10,000$ steps & $5.65(6.85)$ & $4.8(5.85)$ & $9.4(7.05)$ & $77.6(23.15)$ & $17.2(4.7)$ & $20.85(4.65)$ \\
\hline ANOVA Result & $\begin{array}{l}F(1,1313)=3.360 \\
p=.067 \\
\text { eta }^{2}=.003\end{array}$ & $\begin{array}{c}F(1,1313)=.653 \\
p=.419 \\
\text { eta }^{2}=. .000\end{array}$ & $\begin{array}{c}F(1,1313)=.747 \\
p=.388 \\
\text { eta }^{2}=.001\end{array}$ & $\begin{array}{l}F(1,1313)=7.224 \\
\quad p=.007 \\
e^{2} a^{2}=.005\end{array}$ & $\begin{array}{c}F(1,1313)=.306 \\
p=.581 \\
\text { eta }^{2}=.000\end{array}$ & $\begin{array}{l}F(1,1313)=2.170 \\
p=.141 \\
\text { eta }^{2}=.002\end{array}$ \\
\hline
\end{tabular}

*denotes significant improvements in mental health and wellbeing score scores on univariate ANOVA with $\alpha=.05$

outcomes. In terms of the impacts of pre-program subjective reports of physical activity, higher level of self-reported step activity prior to the program was significantly associated with reduced depressive symptoms reporting on the DASS and improvement in wellbeing scores. The self-reported estimate of time spent sitting daily prior to the program was also associated with improved wellbeing scores on the WHO-5 and improvements in sleep related impairment on the PROMIS SRI. Overall, the results highlight the protective effects of age and pre-existing physically active behaviours going into the program.

\section{Discussion}

The participation rates for the activity program over the 50-day period were strong, with the data indicating a very small number of individuals who enrolled in the program failing to record any days of activity $(6.6 \%$ of total cohort) and the majority of the cohort (63.3\%) recording their activity for 40 days or more of the program. The other measure of program engagement of average steps recorded indicated the cohort was particularly active across the program period, logging an average of 12,095 steps, equivalent to an average of $9.7 \mathrm{~km}$ daily. This activity level is markedly greater than the average Australian adult number of steps daily of 7.400 as reported by the Australian Institute of Health and Welfare (2013).

It was notable that comparisons between program completers, dropouts and individuals lost to follow-up showed few differences between the groups on mental health and wellbeing measures at baseline and, even where differences exist, effect size estimates showed they were minimal. This result indicates that in terms of mental health and wellbeing status at commencement, the program completer group is a helpful group to investigate pre-post program changes in mental health and wellbeing outcomes.

Based on our previous findings of mental health improvements after a 100-day physical activity program (Hallam et al., 2018), it was hypothesised that mental health and wellbeing benefits may begin to occur within 50-days of a physical activity program for program completers. The results of this study also indicate a significant improvements in anxiety, wellbeing and sleep related impairment across the 50-day testing window were evident in program completers. Similar trends for improvement were seen with stress measures. The improvements in these measures provide a rounded impression of benefits from more physiological

Table 4 Psychosocial and physical activity predictors of post-program mental health and wellbeing outcomes in program completers $(n=1324)$

\begin{tabular}{|c|c|c|c|c|c|c|c|}
\hline & & \multicolumn{6}{|l|}{ Correlations } \\
\hline & & DASS Depression & DASS Anxiety & DASS Stress & WHO-5 & PROMIS SD & PROMIS SRI \\
\hline Age & & $-.134 * * *$ & $-.206^{* * *}$ & $-.168 * * *$ & $.127 * * *$ & $-246 * * *$ & -.009 \\
\hline Gender & & -.037 & -.014 & .025 & -.025 & .022 & .064 \\
\hline Number Children & & -.057 & -.069 & -.057 & .056 & -.094 & -.009 \\
\hline Team Size & & .002 & -.005 & -.021 & .040 & -.019 & .009 \\
\hline Subjective pre-program steps & & $-.089 *$ & -.084 & -.065 & $.138 * * *$ & -.077 & -.057 \\
\hline Pre-program time sitting & & .044 & .038 & .051 & $-.095^{*}$ & .055 & $.030^{*}$ \\
\hline Regression Statistics & $\begin{array}{l}\mathrm{r}^{2} \\
\text { B } \\
\text { S.E. } \\
\text { p value }\end{array}$ & $\begin{array}{l}.026 \\
9.57 \\
1.52 \\
<.001\end{array}$ & $\begin{array}{l}.048 \\
9.93 \\
1.30 \\
<.001\end{array}$ & $\begin{array}{l}.033 \\
13.72 \\
1.54 \\
<.001\end{array}$ & $\begin{array}{l}.038 \\
63.75 \\
5.07 \\
<.001\end{array}$ & $\begin{array}{l}.066 \\
21.74 \\
1.01 \\
<.001\end{array}$ & $\begin{array}{l}.007 \\
19.919 \\
1.03 \\
.128\end{array}$ \\
\hline
\end{tabular}

\footnotetext{
$*$ denotes significant contributor on regression coefficient at $\alpha=.05, * * *$ denotes significant contributor on regression coefficient at $\alpha=.001$
} 
measures of sleep related impairment to the psychological dimension of anxiety and finally the psychosocial dimension of wellbeing. This aligns with previous research indicating physical activity is an important self-care tool in workers (Syed, 2020). Whether the impacts of the program are related directly to the impacts of exercise cannot be elucidated from the findings of this study which has a naturalistic workplace basis. Improvements across the program could be associated with the social experience, workplace culture changes or any number of other factors. In terms of the possibility that these improvements may reflect impacts of exercise itself, the results are consistent with previous research. One study highlights that significant improvements in mood can be observed over only four months in both a physical activity group (aerobic training three times per week for 45-60 min) and antidepressant treated group (Brenes et al., 2007). Notably, although physical activity can provide similar benefits in improving mood as antidepressant medications in many individuals with mild to moderate symptoms, it has the added advantage of improving cognitive capacity and physical health without the side effects of the medication. Moreover, a Cochrane review indicates that overall, physical activity is as effective as antidepressants for the treatment of depression (Cooney et al., 2013) and there is potential that physical activity may be effective even in antidepressant resistant depressed patients (Mather et al., 2002). These results align with biological findings that mood effects from physical activity occur immediately through the release of endorphins, within four weeks (through changes in the expression of brain derived neurotrophic factor) and with changes in the structure and function of the brain over 10-12 weeks (Cabral et al., 2019). Physical activity positively impacts many body systems. Research now highlights the role of exercise in improving cognition (Tyndall et al., 2018; Sleiman et al., 2016), reducing inflammation (Paolucci et al., 2018) and increasing the expression of brain derived neurotrophic factor (BDNF; Firth et al., 2018) through neurogenesis and synaptogenesis. These effects are posited to account for changes in mental state associated with exercises impact on neurological changes (Kandola et al., 2019; Gujral et al., 2017). These changes are particularly evident in the hippocampus ( $\mathrm{Li}$ et al., 2019) after only eight to ten weeks of exercise (Firth et al., 2018). Anxiety also responds strongly to the effects of exercise with a recent meta-analysis showing a moderate effect size for the intervention (Stubbs et al., 2017b).

It was notable that within the program completer group, we again found no particular importance imbued with the 10,000-step goal itself. This replicates our previous findings of a similar intervention over a 100-day period (Hallam et al., 2018) and a large 1-year follow-up trial in health workers (Lowensteyn et al., 2019). While these findings prove interesting, caution is recommended in abandoning the 10,000-step goal. When considering our earlier reference to the health action process approach (HAPA; Schwarzer, 2008), the setting of concrete goals for both the goal setting and goal pursuit phases may be important. Notably, evidence indicates that the higher the levels of, and engagement in, gamification in physical activity programs, the more effective the program in increasing activity levels (Lier \& Breuer, 2019; Gómez-Carmona et al., 2019).

With increasing evidence (from our own and other published studies) that workplace activity-tracking programs have a positive effect on the mental health of individuals who complete these programs, it becomes increasingly important to better understand who benefits most from these programs. This question formed the final hypotheses that investigated some of the demographic and pre-event selfreported activity level factors that potentially impact relate to post-program mental health and wellbeing outcomes in program completers. The results indicate that younger adults were less likely to demonstrate positive mental health impacts of the program. This may relate to younger adults having greater confidence in their levels of fitness and mental health, younger adults feeling they had less time to engage or a host of other reasons. Alternatively, research indicates that older adults may be more sedentary (Bennie et al., 2015), therefore programs that increase their lower levels of physical activity may lead to larger behavioural changes. There were significant impacts seen in mental health benefits associated with self-reported pre-program step activity levels, specifically associated with an improvement in DASS-21 depression score and improved subjective wellbeing. The self-reported estimate of sedentary behaviour (time spent sitting daily) prior to the program was also associated with improved wellbeing scores on the WHO-5 and improvements in sleep related impairment on the PROMIS SRI post-program. Overall, the results highlight the protective effects of age and pre-existing physically active behaviours going into the program.

The strength of this study was its naturalistic focus and application of the intervention across a range of organizations. There remains a significant efficacy-effectiveness gap between clinical trials and real-world clinical intervention applications. The complexity of organizational culture combined with personal beliefs and behaviours about the value of physical exercise mean real-world applications of interventions with known efficacy from research is vital.

This research is not without its limitations. Whilst the data from fig. 1 highlights that dropout from the program itself was low and most individuals completed the majority of the intervention, many individuals were lost to follow-up in completing their post-program questionnaires. This loss to follow-up of survey completion significantly reduced the sample size of the group that completed the full pre and post testing. Whilst care was taken to fully contrast these groups on the pre-event 
mental health measures, there are risks that the results of the program completers may not represent the results of the larger cohort who were lost to follow-up, a risk that is a significant limitation to the generalisability of the more detailed pre-post intervention outcomes. Due to this risk, definitive comments about mental health outcomes can only be inferred for program completers. The rate of individuals lost to follow-up is similar to drop-out rates reported in previous Australian activity tracking based physical activity challenges (Guertler et al., 2015). Some of the contributing factors for loss to follow up include that the post-event survey occurred after the program was ended and competition winners etc. announced. This likely reduced the motivation of individuals to engage in program related tasks not related to their team outcomes. It was also noted that in this research study, the survey portal was only open for five days post program completion which provided a narrow window for post program survey responding, possibly further minimising feedback on the standard measures. This is a necessary limitation as the mental health outcome measure references the previous week. As the survey component was a completely voluntary addition to this naturalistic program run within workplaces, it was not appropriate to follow-up on scale completion, although this approach may prove beneficial for more comprehensive databases in more controlled trial settings. To address this issue in future research, increased effort in incentivising individuals to complete the questionnaires (noting these are not part of the program itself) is imperative.

The current research also suffers from a lack of objective baseline measures of physical activity and sedentary activity to complement the self-report measures recorded during the program. Because of this, the study was not able to assess changes in actual activity levels to analyse in relation to mental health benefits. It is important to note that the reason for the mental health improvements during the program are therefore not understood and could be associated with the physical activity element of the program or other contextual factors (workplace engagement, enjoyment of the challenge and teamwork or workplace incentives). To overcome this limitation, further programs by the research team will include the Global Physical Activity Questionnaire (Armstrong $\&$ Bull, 2006) in the pre-program assessment.

\section{Conclusions}

This study contributes further to the evidence that workplace based physical activity programs may have a significant impact on mental health in individuals who provide pre and post event mental health data. In this study of 2903 Australian workers, we demonstrate good program engagement in this workplace activity challenge. The sub set of individuals who provided pre and post-program data also highlights significant improvement in anxiety, wellbeing and sleep related impairment over a 50-day period for program completers. Further research is required to find causal underpinnings of this change to establish whether the exercise itself, other program variables or external factors (or a mixture of all these factors) lead to these improvements. This highlights a role for collaboration between industry and researchers in investigating the elements of these programs that improve mental health and wellbeing as the intervention is low cost and low risk. With increasing levels of psychological distress worldwide, particularly in light of the recent COVID-19 pandemic and impacts on the community, improving mental health and wellbeing in workplace settings may be an important focus.

Author Contribution KH contributed to the conduct of the study, data analysis, preparation of manuscript, revisions, and final version approval.

AP contributed to the ethics process, review of manuscript and final version approval.

$\mathrm{KC}$ contributed to data collection and processing, review of manuscript and final version approval.

AG contributed to the ethics process, review of manuscript and final version approval.

SB contributed to the ethics process, collection of data, review of manuscript and final version approval.

Funding This research was funded by Moving Mindz with gratis academic support from Deakin University.

Data Availability The database for this research is available from Deakin University Global Obesity Centre upon reasonable request.

Code Availability Not applicable.

\section{Declarations}

Conflicts of Interest/Competing Interests The authors declare that they have no conflicts of interest with regard to writing this original article.

\section{References}

Abdin, S., Welch, R. K., Byron-Daniel, J., \& Meyrick, J. (2018). The effectiveness of physical activity interventions in improving wellbeing across office-based workplace settings: A systematic review. Public Health, 160, 70-76. https://doi.org/10.1016/j.puhe.2018. 03.029

Aittasalo, M., Rinne, M., Pasanen, M., Kukkonen-Harjula, K., \& Vasankari, T. (2012). Promoting walking among office employees - evaluation of a randomized controlled intervention with pedometers and e-mail messages. BMC Public Health, 12, 403. https:// doi.org/10.1186/1471-2458-12-403

Armstrong, T., \& Bull, F. (2006). Development of the world health organization global physical activity questionnaire (GPAQ). Journal of Public Health, 14(2), 66-70.

Australian Bureau of Statistics. (2018). National Health Survey: First results 2017-2018. Commonwealth of Australia.

Australian Institute of Health and Welfare. (2013). Australian health survey: Physical activity. Catalogue number: 4364.0.55.004. AIHW.

Australian Institute of Health and Welfare (2016). Australian burden of disease study: Impact and causes of illness and death in Australia 
2011. Australian Burden of Disease Study series no. 3. BOD 4. : AIHW.

Bailey, S. K., Haggarty, J., \& Kelly, S. (2016). Global absenteeism and presenteeism in mental health patients referred through primary care. Work, 53, 399-408.

Bennie JA, Pedisic Z, van Uffelen JG, Gale J, Banting LK, Vergeer I, ... Biddle SJ (2015). The descriptive epidemiology of total physical activity, muscle-strengthening exercises and sedentary behaviour among Australian adults-results from the National Nutrition and physical activity survey. BMC Public Health, 16(1), 73.

Booth, F. W., Roberts, C. K., \& Laye, M. J. (2012). Lack of exercise is a major cause of chronic diseases. Comprehensive Physiology, 2, 1143-1211.

Brenes, G. A., Williamson, J. D., Messier, S. P., Rejeski, W. J., Pahor, M., Ip, E., \& Penninx, B. W. (2007). Treatment of minor depression in older adults: A pilot study comparing sertraline and exercise. Aging \& Mental Health, 11, 61-68. https://doi.org/10.1080/ 13607860600736372

Brody B, Burn D. (2015). Should you be aiming for 10,000 steps a day? Available from: http://edition.cnn.com/2015/12/21/health/ ten-thousand-steps-better-health/. Downloaded 12-12-2020.

Brown, T. A., Chorpita, B. F., Korotitsch, W., \& Barlow, D. H. (1997). Psychometric properties of the depression anxiety stress scales (DASS) in clinical samples. Behaviour Research and Therapy, 35, 79-89. https://doi.org/10.1016/S0005-7967(96)00068-X

Cabral, D. F., Rice, J., Morris, T. P., Rundek, T., Pascual-Leone, A., \& Gomes-Osman, J. (2019). Exercise for brain health: An investigation into the underlying mechanisms guided by dose. Neurotherapeutics, 16, 580-599. https://doi.org/10.1007/ s13311-019-00749-w

Cooney, G. M., Dwan, K., Greig, C. A., Lawlor, D. A., Rimer, J., Waugh, F. R., McMurdo, M., \& Mead, G. E. (2013). Exercise for depression. The Cochrane Database of Systematic Reviews, 9, CD004366. https://doi.org/10.1002/14651858.CD004366.pub6

Coutu, M. F., Corbiere, M., Durand, M. J., Nastasia, I., Labrecque, M. E., Berbiche, D., \& Albert, V. (2015). Factors associated with presenteeism and psychological distress using a theory-driven approach. Journal of Occupational and Environmental Medicine, 57, 617-626. https://doi.org/10.1097/JOM.0000000000000459

Crawford, J. R., \& Henry, J. D. (2003). The depression anxiety stress scales (DASS): Normative data and latent structure in a large nonclinical sample. The British Journal of Clinical Psychology, 42, 111-131. https://doi.org/10.1348/014466503321903544

Doran, C. M., \& Kinchin, I. (2019). A review of the economic impact of mental illness. Australian Health Review, 43, 43-48. https:// doi.org/10.1071/AH16115

Ekkekakis, P., \& Murri, M. B. (2017). Exercise as antidepressant treatment: Time for the transition from trials to clinic. General Hospital Psychiatry, 49, A1-A5.

Epton, T., Currie, S., \& Armitage, C. J. (2017). Unique effects of setting goals on behavior change: Systematic review and metaanalysis. Journal of Consulting and Clinical Psychology, 85, 1182-1198. https://doi.org/10.1037/ccp0000260

Field, A. (2018). Discovering statistics using IBM SPSS statistics. Sage Publishing.

Firth, J., Stubbs, B., Vancampfort, D., et al. (2018). Effects of aerobic exercise on hippocampal volume in humans: A systematic review and meta-analysis. Neuroimage., 166, 230-238. https:// doi.org/10.1016/j.neuroimage.2017.11.007

Freak-Poli, R. L., Wolfe, R., Wong, E., \& Peeters, A. (2014). Change in well-being amongst participants in a four-month pedometerbased workplace health program. BMC Public Health, 14, 953. https://doi.org/10.1186/1471-2458-14-953

Gerber, M., Jonsdottir, I. H., Lindwall, M., \& Ahlborg, G., Jr. (2014). Physical activity in employees with differing occupational stress and mental health profiles: A latent profile analysis. Psychology of Sport and Exercise, 15, 649-658. https://doi.org/10.1016/j. psychsport.2014.07.012

Glance DG, Ooi E, Berman YE, Glance CF, Barrett HR (2016) Impact of a digital activity tracker-based workplace activity program on health and wellbeing. In Proceedings of the 6th International Conference on Digital Health, 37-41. https://doi. org/10.1145/2896338.2896345.

Gómez-Carmona O, Casado-Mansilla D, García-Zubía J (2019) Opportunities and Programs of Technology-Based Interventions to Increase Health-Awareness in the Workplace. In Vega-Barbas and Seoane (Eds.), Transforming Ergonomics with Personalized Health and Intelligent Workplaces, 25, 33. https://doi.org/10. 3233/978-1-61499-973-7-33.

Guertler, D., Vandelanotte, C., Kirwan, M., \& Duncan, M. J. (2015). Engagement and non-usage attrition with a free physical activity promotion program: The case of 10,000 steps Australia. Journal of Medical Internet Research, 17, e176.

Gujral, S., Aizenstein, H., Reynolds, C. F., III, Butters, M. A., \& Erickson, K. I. (2017). Exercise effects on depression: possible neural mechanisms. General Hospital Psychiatry, 49, 2-10. https://doi.org/10.1016/j.genhosppsych.2017.04.012

Hall, T., Krahn, G. L., Horner-Johnson, W., \& Lamb, G. (2011). Examining functional content in widely used health-related quality of life scales. Rehabilitation Psychology, 56, 94-99. https://doi.org/10.1037/a0023054

Hallam, K. T., Bilsborough, S., \& de Courten, M. (2018). "Happy feet": Evaluating the benefits of a 100 -day 10,000 step program on mental health and wellbeing. BMC Psychiatry, 18, 19. https://doi.org/10.1186/s12888-018-1609-y

Harding, J., Freak-Poli, R. L. A., Backholer, K., \& Peeters, A. (2013). Change in health-related quality of life amongst participants in a 4-month pedometer-based workplace health program. Journal of Physical Activity and Health, 10, 533-543. https://doi.org/ 10.1186/1471-2458-14-953

Hayes, S. C., Newton, R. U., Spence, R. R., \& Galvão, D. A. (2019). The exercise and sports science Australia position statement: Exercise medicine in cancer management. Journal of Science and Medicine in Sport, 22(11), 1175-1199.

Hilton, M. F., \& Whiteford, H. A. (2010). Associations between psychological distress, workplace accidents, workplace failures and workplace successes. International Archives of Occupational and Environmental Health, 83, 923-933. https://doi.org/ 10.1007/s00420-010-0555-X

Holden, L., Scuffham, P. A., Hilton, M. F., Ware, R. S., Vecchio, N., \& Whiteford, H. A. (2011). Which health conditions impact on productivity in working Australians? Journal of Occupational and Environmental Medicine, 53, 253-257. https://doi.org/10.1097/ JOM.0b013e31820d1007

Johnson, D., Deterding, S., Kuhn, K. A., Staneva, A., Stoyanov, S., \& Hides, L. (2016). Gamification for health and wellbeing: A systematic review of the literature. Internet Interventions, 6, 89-106. https://doi.org/10.1016/j.invent.2016.10.002

Joyce, S., Modini, M., Christensen, H., Mykletun, A., Bryant, R., Mitchell, P. B., \& Harvey, S. B. (2016). Workplace interventions for common mental disorders: A systematic meta-review. Psychological Medicine, 46, 683-697. https://doi.org/10.1017/S0033 291715002408

Kandola, A., Ashdown-Franks, G., Hendrikse, J., Sabiston, C. M., \& Stubbs, B. (2019). Physical activity and depression: Towards understanding the antidepressant mechanisms of physical activity. Neuroscience \& Biobehavioral Reviews, 107, 525-539. https:// doi.org/10.1016/j.neubiorev.2019.09.040

KPMG and Mental Health Australia (2018). Investing to save: The Economic Benefits for Australia of Investment in Mental Health Reform. Available from https://mhaustralia.org/sites/default/files/ 
docs/investing_to_save_may_2018_-_kpmg_mental_health_austr alia.pdf Accessed 14th June 2020.

Li, A., Yau, S. Y., Machado, S., Wang, P., Yuan, T. F., \& So, K. F. (2019). Enhancement of hippocampal plasticity by physical exercise as a polypill for stress and depression: A review. $C N S \&$ Neurological Disorders-Drug Targets, 18, 294-306. https://doi. org/10.2174/1871527318666190308102804

Lier, L. M., \& Breuer, C. (2019). The motivating power of gamification. International Journal of Workplace Health Management, 13, 1-15. https://doi.org/10.1108/IJWHM-04-2019-0055

Lovibond, S. H., \& Lovibond, P. F. (1995). Manual for the depression anxiety stress scales. The Psychology Foundation of Australia.

Lowensteyn, I., Berberian, V., Belisle, P., DaCosta, D., Joseph, L., \& Grover, S. A. (2018). The measurable benefits of a workplace wellness program in Canada. Journal of Occupational and Environmental Medicine, 60, 211-216. https://doi.org/10.1097/JOM. 0000000000001240

Lowensteyn, I., Berberian, V., Berger, C., Da Costa, D., Joseph, L., \& Grover, S. A. (2019). The sustainability of a workplace wellness program that incorporates gamification principles: Participant engagement and health benefits after 2 years. American Journal of Health Promotion, 33, 850-858. https://doi.org/10.1177/08901 17118823165

Mather, A. S., Rodriguez, C., Guthrie, M. F., McHarg, A. M., Reid, I. C., \& McMurdo, M. E. (2002). Effects of exercise on depressive symptoms in older adults with poorly responsive depressive disorder: Randomised controlled trial. The British Journal of Psychiatry, 180, 411-415. https://doi.org/10.1192/bjp.180.5.411

Paolucci, E. M., Loukov, D., Bowdish, D. M., \& Heisz, J. J. (2018). Exercise reduces depression and inflammation but intensity matters. Biological Psychology, 133, 79-84.

Perneger, T. V. (1998). What's wrong with Bonferroni adjustments. BMJ, 316(7139), 1236-1238.

Productivity Commission (2019). Mental Health, Draft Report, Canberra. Retrieved from https://www.pc.gov.au/inquiries/compl eted/mental-health/draft/mental-health-draft-overview.pdf on 14th June 2020.

Reiner, M., Niermann, C., Jekauc, D., \& Woll, A. (2013). Long-term health benefits of physical activity-a systematic review of longitudinal studies. BMC Public Health, 13, 1-9. https://doi.org/10. 1186/1471-2458-13-813

Robertson, I. T., \& Cooper, C. L. (2010). Full engagement: The integration of employee engagement and psychological well-being. Leadership \& Organization Development Journal, 31, 324-336.

Salmon, P. (2001). Effects of physical exercise on anxiety, depression, and sensitivity to stress: A unifying theory. Clinical Psychology Review, 21, 33-61. https://doi.org/10.1016/s02727358(99)00032-x

Sanders, J. P., Loveday, A., Pearson, N., Edwardson, C., Yates, T., Biddle, S. J., \& Esliger, D. W. (2016). Devices for self-monitoring sedentary time or physical activity: A scoping review. Journal of Medical Internet Research, 18, e90. https://doi.org/ 10.2196/jmir.5373

Schuch, F. B., Vancampfort, D., Richards, J., Rosenbaum, S., Ward, P. B., \& Stubbs, B. (2016). Exercise as a treatment for depression: A meta-analysis adjusting for publication bias. Journal of Psychiatric Research, 77, 42-51. https://doi.org/10.1016/j. jpsychires.2016.02.023

Schwarzer, R. (2008). Modeling health behavior change: How to predict and modify the adoption and maintenance of health behaviors. Applied Psychology, 57, 1-29. https://doi.org/10. 1111/j.1464-0597.2007.00325.x
Sleiman SF, Henry J, Al-Haddad R, El Hayek L, Abou Haidar E, Stringer T, Ulja D, Karuppagounder SS, Holson EB, Ratan RR, Ninan I, Chao MV (2016) Exercise promotes the expression of brain derived neurotrophic factor (BDNF) through the action of the ketone body $\beta$-hydroxybutyrate. eLife, 5: e15092. https://doi. org/10.7554/eLife.15092.

Sounan, C., Lavoie-Tremblay, M., Martin, K., Trudel, J., Lavigne, G., Lowensteyn, I., \& Grover, S. A. (2013). Impact of a pedometer-based physical activity program on behavioral, biomedical, anthropometric and psychological outcomes in hospital employees: An interventional study. Clin Health Promot, 3, 5-11. https://doi.org/10.29102/clinhp.13002

Strainge, L., Sullivan, M. C., Blackmon, J. E., Cruess, S. E., Wheeler, D., \& Cruess, D. G. (2019). PROMIS®-assessed sleep problems and physical health symptoms in adult psychiatric inpatients. Health Psychology, 38(5), 376.

Stubbs, B., Vancampfort, D., Rosenbaum, S., et al. (2017a). An examination of the anxiolytic effects of exercise for people with anxiety and stress-related disorders: A meta-analysis. Psychiatry Research, 249, 102-108. https://doi.org/10.1016/j.psychres. 2016.12.020

Stubbs, B., Koyanagi, A., Hallgren, M., et al. (2017b). Physical activity and anxiety: A perspective from the world health survey. Journal of Affective Disorders, 208, 545-552. https://doi. org/10.1016/j.jad.2016.10.028

Syed, I. U. (2020). Diet, physical activity, and emotional health: What works, what doesn't, and why we need integrated solutions for total worker health. BMC Public Health, 20(152), 1-9. https://doi.org/10.1186/s12889-020-8288-6

Topp, C. W., Østergaard, S. D., Søndergaard, S., \& Bech, P. (2015). The WHO-5 well-being index: A systematic review of the literature. Psychotherapy and Psychosomatics, 84, 167-176. https:// doi.org/10.1159/000376585

Tyndall, A. V., Clark, C. M., Anderson, T. J., Hogan, D. B., Hill, M. D., Longman, R. S., \& Poulin, M. J. (2018). Protective effects of exercise on cognition and brain health in older adults. Exercise and Sport Sciences Reviews, 46, 215-223. https://doi.org/10. 1249/JES.0000000000000161

Wattanapisit, A., \& Thanamee, S. (2017). Evidence behind 10,000 steps walking. Journal of Health Research, 31(3), 241-248.

World Health Organization (1994). Global strategy on occupational health for all: The way to health at work. Recommendation of the second meeting of the WHO Collaborating Centres in Occupational Health, 11-14 October 1994, Beijing, China.

World Health Organization (2011). Global burden of mental disorders and the need for a comprehensive, coordinated response from health and social sectors at the country level: Report by the Secretariat. Retrieved from https://apps.who.int/iris/handle/ 10665/23741 (Accessed 23/11/20).

Yap, Y., Slavish, D. C., Taylor, D. J., Bei, B., \& Wiley, J. F. (2020). $\mathrm{Bi}$-directional relations between stress and self-reported and actigraphy-assessed sleep: A daily intensive longitudinal study. Sleep, 43(3), zsz250.

Yu, L., Buysse, D. J., Germain, A., Moul, D. E., Stover, A., Dodds, N. E., Johnston, K. L., \& Pilkonis, P. A. (2011). Development of short forms from the PROMIS ${ }^{\mathrm{TM}}$ sleep disturbance and sleeprelated impairment item banks. Behavioral Sleep Medicine, 10(1), 6-24. https://doi.org/10.1080/15402002.2012.636266

Publisher's Note Springer Nature remains neutral with regard to jurisdictional claims in published maps and institutional affiliations. 